\title{
Efetividade dos programas de exercícios físicos sobre as respostas cardiorrespiratórias em mulheres no período gestacional e pós parto
}

Effectiveness of physical exercise programs on cardiorespiratory responses in women during pregnancy and postpartum

Efectividad de los programas de ejercicio físico sobre las respuestas cardiorrespiratorias en mujeres durante el embarazo y el posparto

Gustavo Baroni Araujo ORCID: https://orcid.org/0000-0002-3162-7477 Universidade Estadual de Londrina, Brasil E-mail: gustavobaroni13@hotmail

Célio Pereira de Sousa Júnior ORCID: https://orcid.org/0000-0003-0726-0668 Universidade Federal do Pará, Brasil E-mail: academicocelio@gmail.com

Yasmin Emanuelly Leal Araujo ORCID: https://orcid.org/0000-0002-7550-636X Universidade Federal do Piauí, Brasil

E-mail: nutriyasminemanuelly@gmail.com

Fábio José Antônio da Silva

ORCID: https://orcid.org/0000-0002-5881-6438

Universidade Estadual de Londrina, Brasil E-mail: fjas81@hotmail.com

Bruno Abílio da Silva Machado ORCID: https://orcid.org/0000-0003-1759-0206 Centro Universitário Mauricio de Nassau, Brasil

E-mail: bruno.smachado@ufpe.br

Joelma Maria dos Santos da Silva Apolinário ORCID: https://orcid.org/0000-0001-9521-9432 Faculdade Maurício de Nassau, Brasil E-mail: jo.silva00@hotmail.com

Glenda Suellen Matos Cruz ORCID: https://orcid.org/0000-0003-1933-1937 Faculdade Dom Pedro II, Brasil

E-mail: gl.suellen@hotmail.com

Eleticia da Silva Carvalho

ORCID: https://orcid.org/0000-0003-4351-2789 Faculdade Dom Pedro II, Brasil E-mail: eleticiasc13@gmail.com Edileide de Jesus Freire

ORCID: https://orcid.org/0000-0001-5636-6894 Faculdade Dom Pedro II, Brasil E-mail: sedileide816@gmail.com

Ingrid Mikaela Moreira de Oliveira ORCID: https://orcid.org/0000-0002-8901-362X Universidade Estadual do Ceará, Brasil E-mail: ingrid_lattes@hotmail.com Janaina Cesário Araujo ORCID: https://orcid.org/0000-0002-3394-2593

Universidade Estadual da Paraíba, Brasil E-mail: janainacesario07@gmail.com

Emmanuella Costa de Azevedo Mello ORCID: https://orcid.org/0000-0001-9747-2992 Universidade Federal da Paraíba, Brasil

E-mail: emmanuellaazevedo@ hotmail.com

João Paulo Barros Ibiapina

ORCID: https://orcid.org/0000-0003-1791-7774 Universidade Federal do Piauí, Brasil E-mail:jp072001@gmail.com 


\author{
Barbara Rodrigues Ferreira \\ ORCID: https://orcid.org/0000-0003-1154-4364 \\ Universidade Federal do Pará, Brasil \\ E-mail:barbararodriguesatm22@gmail.com \\ Wesley Romário Dias Martins \\ ORCID: https://orcid.org/0000-0002-9270-056X \\ Universidade Estadual do Piauí, Brasil \\ E-mail: wesleyrdm_enf@outlook.com.br \\ Karla Karolaine Silva de Carvalho \\ ORCID: https://orcid.org/0000-0003-3712-8780 \\ Centro Universitário UNIFAVIP, Brasil \\ E-mail: karlakarolaine5@gmail.com \\ Aline Ribeiro Ferreira \\ ORCID: https://orcid.org/0000-0001-5031-2422 \\ Faculdade Integrada de Brasília, Brasil \\ E-mail: aline_ferreira94@outlook.com
}

\begin{abstract}
Resumo
As modificações fisiológicas que ocorrem no decorrer da vida das mulheres no período gestacional e pós parto já são bem evidenciadas na literatura cientifica. Sabe-se que a prática de atividade física pode ser um importante fator de proteção frente as condições observadas nestes marcos na saúde da mulher. O objetivo deste trabalho foi discutir a efetividade dos programas de exercícios físicos sobre as respostas cardiorrespiratórias em mulheres no período gestacional e pós parto. Trata-se de uma revisão de literatura. A busca consistiu nas bases de dados Scientific Electronic Library Online (SciELO) e Centro Latino-Americano e do Caribe de Informação em Ciências da Saúde (Bireme). Os artigos foram selecionados a partir das seguintes palavras-chave em língua portuguesa: exercício de força, gestação, pós parto. Foram selecionados apenas artigos publicados de 2012 a 2020, estudos de revisão de literatura e apenas da língua portuguesa e língua inglesa. No total, 7 estudos fizeram parte desta revisão. As melhorias cardiorrespiratórias em virtude do exercício físico é positivo para gestante uma vez que contribui para a saúde da mulher e do bebê, bem como as melhorias cardiorrespiratórias para mulheres na fase pós parto, considerando as particularidades deste marco, diminuindo agravos em saúde na fase da maternidade. Ressalta-se a necessidade dos profissionais da saúde, em especial, profissionais de educação física busquem conhecimentos a respeito das prescrições, orientações e contraindicações na realização de atividade física para essa população, para que a intervenção seja realizada de forma eficaz e segura garantindo a saúde da mulher.
\end{abstract}

Palavras-chave: Saúde da mulher; Exercícios físicos; Gestantes; Pós parto.

\begin{abstract}
The physiological changes that occur during the life of women during pregnancy and postpartum are already well evidenced in the scientific literature. It is known that the practice of physical activity can be an important protective factor against the conditions observed in these milestones in women's health. The objective of this study was to discuss the effectiveness of physical exercise programs on cardiorespiratory responses in women during pregnancy and postpartum period. This is a literature review. The search consisted of the Scientific Electronic Library Online (SciELO) and the Latin American and Caribbean Center for Health Sciences Information (Bireme). The articles were selected based on the following keywords in Portuguese: strength exercise, pregnancy, postpartum. Only articles published from 2012 to 2020, literature review studies and only in Portuguese and English were selected. In total, 7 studies were part of this review. Cardiorespiratory improvements due to physical exercise are positive for pregnant women as it contributes to the health of women and babies, as well as cardiorespiratory improvements for women in the postpartum phase, considering the particularities of this milestone, reducing health problems in the maternity. The need for health professionals, in particular physical education professionals, to seek knowledge about the prescriptions, guidelines and contraindications in performing physical activity for this population is highlighted, so that the intervention is carried out effectively and safely, ensuring health of the woman.
\end{abstract}

Keywords: Women's health; Physical exercises; Pregnant women; Post childbirth.

\title{
Resumen
}

Los cambios fisiológicos que ocurren durante la vida de la mujer durante el embarazo y el posparto ya están bien evidenciados en la literatura científica. Se sabe que la práctica de actividad física puede ser un factor protector importante frente a las condiciones que se observan en estos hitos en la salud de la mujer. El objetivo de este estudio fue discutir la efectividad de los programas de ejercicio físico sobre las respuestas cardiorrespiratorias en mujeres durante el embarazo y el posparto. Ésta es una revisión de la literatura. La búsqueda consistió en la Scientific Electronic Library Online (SciELO) y el Centro Latinoamericano y del Caribe de Información en Ciencias de la Salud (Bireme). Los artículos fueron seleccionados en base a las siguientes palabras clave en portugués: ejercicio de fuerza, embarazo, posparto. Solo se seleccionaron artículos publicados de 2012 a 2020, estudios de revisión de literatura y solo en portugués e inglés. En total, siete estudios formaron parte de esta revisión. Las mejoras cardiorrespiratorias debidas al ejercicio físico son positivas para la gestante ya que contribuye a la salud de la mujer y el bebé, así como 
mejoras cardiorrespiratorias para la mujer en la fase posparto, considerando las particularidades de este hito, reduciendo los problemas de salud en la maternidad. Se destaca la necesidad de que los profesionales de la salud, en particular los profesionales de la educación física, busquen conocimiento sobre las prescripciones, pautas y contraindicaciones en la realización de actividad física para esta población, de manera que la intervención se lleve a cabo de manera efectiva y segura, velando por la salud de la mujer.

Palabras clave: La salud de la mujer; Ejercicios físicos; Mujeres embarazadas; Post parto.

\section{Introdução}

Já são bem estabelecidas as modificações fisiológicas que ocorrem no decorrer da vida das mulheres, principalmente no período gestacional. Sabe-se que as alterações envolvem questões biológicas, morfológicas e psicossociais. Entre as principais consequências do período gestacional tem-se, no primeiro trimestre as adaptações da gestante à sua nova condição. Este começo da gravidez pode provocar grandes oscilações emocionais e maior sensibilidade nos seios, sono e cansaço, além de enjoos (American College of Obstetricians and Gynecologists, 1994).

A pratica regular de atividade física bem como a adoção de hábitos saudáveis são importantes fatores de prevenção ao desenvolvimento de doenças crônicas não transmissíveis, como Diabetes tipo II, Hipertensão, Obesidade entre outras. Entretanto, sabe-se que a realização de programas de exercícios físicos tanto na fase gestacional quanto no período pós parto pode ser bastante benéfico para a saúde da mulher, mantendo suas capacidades físicas, como a capacidade cardiorrespiratória, de força e dos demais componentes da aptidão física (Coutinho et al., 2014).

A recomendação da prática de atividade física do tipo cardiorrespiratórias e de força como por exemplo, a realização de caminhadas e musculação tem sido frequentemente recomendada por profissionais da saúde (Lehnen et al., 2019). A Organização Mundial da Saúde recomenda a prática regular de atividade física de intensidade moderada a vigorosa por pelo menos 150 minutos semanais. Diversos estudos longitudinais têm evidenciado que a adoção deste habito pode contribuir a uma melhor qualidade de vida e longevidade (Felix \& oliveira, 2021).

Neste sentido, como já evidenciado, é indicado que mulheres gestantes e em período pós parto realizem de forma regular exercícios de força e cardiorrespiratórios com o propósito de diminuir os prejuízos causados por ambas as fases (Rocha et al., 2019). Entretanto, existem importantes considerações a serem abordadas no que diz respeito a prescrição como número de exercícios, intensidade do exercício duração, e alguns cuidados específicos a respeito da orientação, como correções, número de vezes na semana e particularidade do caso, além dos cuidados de forma geral com essa população nestes períodos (Freitas, 2017).

Dada a discussão, considera-se a temática de grande relevância para se discutir a efetividade dos programas de exercício físico sobre as respostas cardiovasculares em gestantes e mulheres no pós parto, bem como assimilar a importância da atividade física para a diminuição de prejuízos à saúde e a necessidade de conhecimentos dos profissionais de Educação Física no âmbito da saúde da mulher proporcionando maior entendimento sobre as particularidades da fase gestacional e exercícios físicos sobre as respostas cardiorrespiratórias durante a prática de atividade física.

\section{Metodologia}

Trata-se de um estudo de revisão retrospectiva, bibliográfica e exploratória, realizado entre os meses de outubro e de novembro de 2021, com abordagem descritiva, na base de dados nas bases de dados Scientific Electronic Library Online (SciELO) e Centro Latino-Americano e do Caribe de Informação em Ciências da Saúde (Bireme). Os artigos foram selecionados a partir das seguintes palavras-chave em língua portuguesa: exercício físico, gestação, pós-parto. 
A confecção do trabalho seguiu as seguintes etapas: 1-Elaboração de pergunta norteadora; 2-Busca ou amostragem na literatura; 3-Coleta de dados; 4-Análise crítica dos estudos incluídos; 5-Discussão dos resultados; e 6-Apresentação dos resultados obtidos com o objetivo de responder à questão norteadora (Estrela, 2018) “ .

Os critérios de inclusão foram artigos completos publicados entre o período de janeiro de 2012 a dezembro de 2020 identificados em outubro de 2021. Somente foram incluídos artigos que tivessem alguma relação com exercícios físico e atividade física em mulheres gestantes ou em pós parto. Foram excluídos resumos (simples e expandidos) apresentados em eventos científicos e resumos (simples ou expandidos) publicados em anais de eventos científicos.

Posteriormente a aplicação dos critérios de inclusão e exclusão, os trabalhos foram selecionados e avaliados por título e resumo com o propósito de direcionar a temática para este estudo. Esta avaliação foi realizada por um pesquisador. Para os casos em que a leitura do resumo não fosse suficiente para definir a inclusão do artigo, foram considerados os demais critérios e a leitura na íntegra. Os dados de interesse dos trabalhos selecionados foram extraídos e registrados em planilha padronizada, que continha as principais informações sobre cada artigo.

A elegibilidade de cada estudo foi determinada pela leitura na íntegra e sua identificação é demonstrada no fluxograma da Figura 1.

Figura 1: Fluxograma do processo de identificação e seleção de artigos.
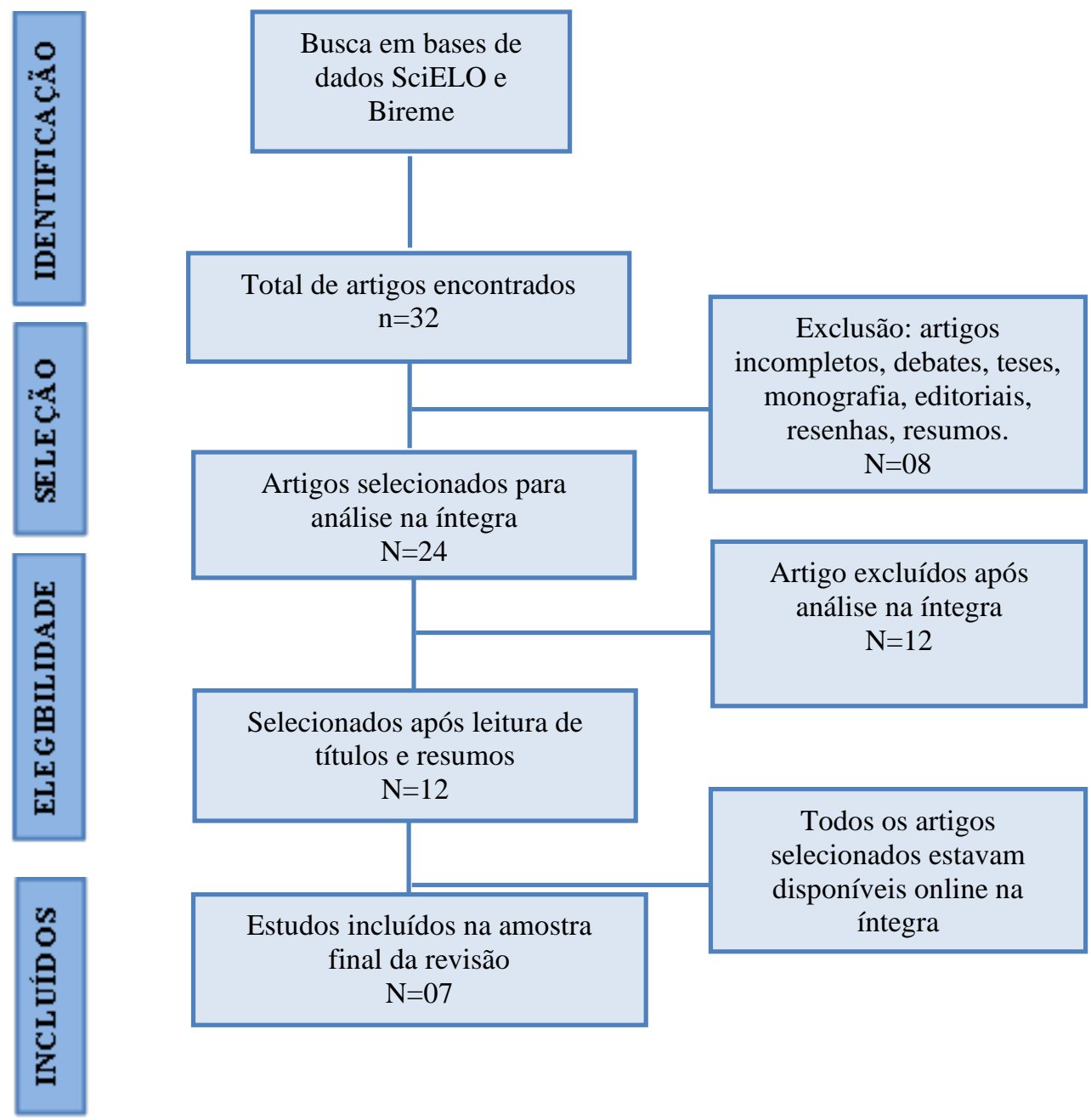

Fonte: Autores (2021). 


\section{Resultados e Discussão}

Inicialmente, foram encontrados 32 artigos publicados nas bases de dados SciELO e Bireme. Foram aplicados os critérios de inclusão e exclusão e selecionados 24 artigos para a análise na íntegra, no entanto, após analisados os critérios de elegibilidade, apenas 7 artigos foram aprovados para este trabalho. Todos os artigos selecionados estavam relacionados a prática de exercício físico na gestação ou no pós parto, conforme apresentado no quadro 1.

Quadro 1: Caracterização dos estudos incluídos na revisão segundo número, autores e ano, título, objetivos, e resultados e considerações.

\begin{tabular}{|c|c|c|c|c|}
\hline $\mathbf{N}^{\mathbf{o}}$ & Autor e ano & Título & Objetivo & Resultados e considerações \\
\hline 1 & $\begin{array}{l}\text { Nascimento et al. } \\
2014\end{array}$ & $\begin{array}{l}\text { Recomendações } \\
\text { para a prática de } \\
\text { exercício físico na } \\
\text { gravidez: uma } \\
\text { revisão crítica da } \\
\quad \text { literatura }\end{array}$ & $\begin{array}{l}\text { Informar e disseminar o conhecimento } \\
\text { entre profissionais de saúde, que } \\
\text { assistem às gestantes no Brasil, das } \\
\text { atuais recomendações sobre exercício } \\
\text { físico durante a gestação, baseadas nas } \\
\text { evidências científicas disponíveis. }\end{array}$ & $\begin{array}{l}\text { Recomenda-se a prática regular de exercícios físicos nas } \\
\text { modalidades exercícios aeróbicos, treinamento de } \\
\text { resistência muscular, alongamento e exercícios do assoalho } \\
\text { pélvico, frequência, intensidade e duração dos exercícios } \\
\text { para cada trimestre gestacional. Indica-se a realização de } \\
\text { exercícios físicos tanto para as gestantes de baixo risco } \\
\text { quanto para populações especiais de mulheres, como atletas, } \\
\text { hipertensas, diabéticas e obesas. }\end{array}$ \\
\hline 2 & Tebas et al. 2012 & $\begin{array}{l}\text { Gestação e exercício } \\
\text { físico: } \\
\text { recomendações, } \\
\text { cuidados e } \\
\text { prescrição. }\end{array}$ & $\begin{array}{l}\text { Avaliar os efeitos do treinamento físico } \\
\text { durante a gestação na evolução } \\
\text { ponderal, circunferência abdominal, } \\
\text { glicemia e colesterolemia de filhotes } \\
\text { adultos submetidos à desnutrição } \\
\text { perinatal }\end{array}$ & $\begin{array}{l}\text { O treinamento físico durante a gestação atenua os efeitos da } \\
\text { desnutrição perinatal sobre alguns indicadores } \\
\text { murinométricos e bioquímicos }\end{array}$ \\
\hline 3 & Baldo et al. 2020 & $\begin{array}{l}\text { Vulnerabilidades da } \\
\text { população idosa } \\
\text { durante a pandemia } \\
\text { pelo novo } \\
\text { coronavírus }\end{array}$ & $\begin{array}{l}\text { Discutir os benefícios da atividade física } \\
\text { durante a gestação e as } \\
\text { diretrizes que norteiam a prescrição } \\
\text { segura para essa população }\end{array}$ & $\begin{array}{l}\text { Foi demonstrado que os exercícios físicos trazem inúmeros } \\
\text { benefícios, tais como: manutenção do peso, alívio de dores } \\
\text { posturais, melhora de função cardiovascular, auxílio no } \\
\text { equilíbrio glicêmico, prevenção de doenças ou demais } \\
\text { complicações, além de melhorar quadros de estresse e } \\
\text { ansiedade }\end{array}$ \\
\hline 4 & $\begin{array}{l}\text { Rebesco et al } \\
2016\end{array}$ & $\begin{array}{l}\text { Ação do exercício } \\
\text { físico na gestação: } \\
\text { um estudo de } \\
\text { revisão }\end{array}$ & $\begin{array}{l}\text { Realizar uma revisão bibliográfica e } \\
\text { analisar informações pertinentes sobre a } \\
\text { ação do exercício físico, a respeito dos } \\
\text { riscos e benefícios durante a gestação. }\end{array}$ & $\begin{array}{l}\text { As seguintes recomendações foram semelhantes na maioria } \\
\text { dos trabalhos: que o exercício físico na gestação contribui } \\
\text { para a manutenção da aptidão física, diminui sintomas } \\
\text { gravídicos, melhora o controle ponderal, diminuí a tensão no } \\
\text { parto, ajuda na recuperação no pós-parto, bem como } \\
\text { prevenção de algumas desordens, como o diabetes } \\
\text { gestacional. }\end{array}$ \\
\hline 5 & Silva et al. 2020 & $\begin{array}{l}\text { Exercício físico } \\
\text { durante a gestação: } \\
\text { os benefícios para } \\
\text { mãe e o bebê }\end{array}$ & $\begin{array}{l}\text { Discutir a importância do exercício } \\
\text { físico durante a gestação: os benefícios } \\
\text { para a mãe e o bebê. }\end{array}$ & $\begin{array}{l}\text { O exercício bom para grávida é aquele que a faz se sentir } \\
\text { bem e confortável. A escolha da modalidade deve partir da } \\
\text { gestante, no entanto é preciso que tenha a liberação médica } \\
\text { e respeite as condições provenientes da gravidez. O } \\
\text { profissional de educação física deve estar preparado e } \\
\text { conhecer todas as alterações e sintomas desse período, para } \\
\text { que a prática dos exercícios seja sempre benéfica para a mãe } \\
\text { e o bebê. }\end{array}$ \\
\hline 6 & Silva et al. 2017 & $\begin{array}{l}\text { Musculação para } \\
\text { gestantes: Entre } \\
\text { efeitos e indicações }\end{array}$ & $\begin{array}{l}\text { Ajudar outros profissionais de educação } \\
\text { física a } \\
\text { trabalharem com gestantes de uma } \\
\text { forma segura e significativa }\end{array}$ & $\begin{array}{l}\text { Ressalta-se a importância da musculação durante a gestação } \\
\text { e os } \\
\text { benefícios da prática. }\end{array}$ \\
\hline 7 & $\begin{array}{l}\text { Bonfim et al. } \\
2016\end{array}$ & $\begin{array}{l}\text { A Influência do } \\
\text { exercício físico na } \\
\text { gravidez e no } \\
\text { momento do parto }\end{array}$ & $\begin{array}{l}\text { Comparar de forma clara os benefícios } \\
\text { que o exercício físico trás para a vida da } \\
\text { gestante ativa durante aproximadamente } \\
40 \text { semanas, com a finalidade de } \\
\text { incentivar a essa população e } \\
\text { profissionais da área da saúde uma } \\
\text { prática de exercícios físicos desde que } \\
\text { estruturados e orientados }\end{array}$ & $\begin{array}{l}\text { O exercício físico ajuda a reduzir o inchaço, a melhorar a } \\
\text { circulação sanguínea, amplia o equilíbrio muscular, alivia os } \\
\text { desconfortos intestinais, diminui as câimbras, fortalece e } \\
\text { facilita na recuperação pós-parto }\end{array}$ \\
\hline
\end{tabular}

Fonte: Autores (2021).

Os principais resultados obtidos dos sete artigos selecionados foram explicitados no Quadro 1, com avaliação do autor responsável de acordo com as informações levantadas. 
Em relação ao ano de publicação dos artigos originais analisados, um trabalho $(14,2 \%)$ foi publicado em 2012, um trabalho $(14,2 \%)$ foi publicado em 2014 , dois trabalhos $(28,4 \%)$ foram apresentados em 2016 , um trabalho $(14,2 \%)$ foi publicado em 2017 e dois $(28,4 \%)$ publicados em 2020.

De acordo com as informações encontras na literatura, os tipos de exercícios que apresentaram melhores resultados para as melhorias nas respostas cardiorrespiratórias em gravidas e mulheres pós parto foram os exercícios de força, caracterizados por atuarem de forma efetiva no fortalecimento da musculatura e contribuir para a capacidade cardiorrespiratória, sendo assim, esse tipo de programa de atividade física se mostra eficaz para a melhoria desta capacidade física.

A prática de atividade física se tornou um comportamento primordial para a proteção e promoção da saúde nas populações contemporâneas, pois o estilo de vida adotado atualmente inclui o aumento do sedentarismo e de hábitos alimentares inadequados que podem acarretar o excesso de peso. Estes são alguns dos principais fatores de risco para o desenvolvimento das doenças crônicas não transmissíveis principais causas de morbidade e mortalidade no Brasil e no mundo (Organização Pan-Americana de Saúde, 2018).

Para mulheres gestantes, os benefícios da atividade física são destacados nas diretrizes canadense para gestantes, que tem como objetivo evitar o excesso de peso, o desenvolvimento de diabetes gestacional e diminuir as chances da depressão pós-parto (Oliveira \& Mattos, 2016). Um dado importante apresentado no documento que chama a atenção é que a atividade física aumenta as chances de sucesso do parto normal diminuindo as chances de parto por cesárea.

Os benefícios dos exercícios de força na gestação envolvem questões biológicas e psicossociais. Entende-se que a prática de exercícios de força pode ser melhor tolerada na fase gestacional em relação aos exercícios do tipo aeróbico por serem responsáveis por menores estresses cardiovasculares. Entre as principais respostas cardiovasculares na gestação, tem-se o aumento da pressão intrauterina onde passa a existir um aumento do volume diastólico final e um pequeno aumento da massa e da parede do ventrículo esquerdo (Silva et al., 2020). Além disso, as mudanças anatômicas e fisiológicas que ocorrem no período da gravidez podem afetar o sistema musculoesquelético no repouso e durante o exercício.

Os efeitos observados após a prática regular de exercícios de força podem ocorrer de forma aguda ou crônica. Recomenda-se desde que a gestante não apresente complicações físicas ou uma gravidez de risco pelo menos três vezes por semana a realização deste treinamento. Os treinamentos de força, ou exercícios resistidos são um conjunto de exercícios que tem como objetivo o aumento da força muscular e da resistência para as atividades da vida diária, além de ser considerado uma forma eficiente da redução da gordura corporal além de favorecer a resistência anaeróbia (Pereira \& Aguiar, 2016). De forma geral, estes exercícios são compostos por exercícios que compõem glúteos, quadríceps, abdutores, adutores, etc.

A efetividade do treinamento de força para gestantes sobre as respostas cardiovasculares é positiva considerando as alterações que ocorrem durante a gravidez. O aumento da barriga, a pressão intrauterina, alterações do volume sistólico, diastólico e debito cardíaco são importantes marcadores fisiológicos que necessitam de maior atenção na pratica do treinamento, tendo em vista que os exercícios resistidos irão, posteriormente, causar a hipotensão pós exercício, melhor os indicies de tolerância a glicose tendo em vista a possibilidade da mulher desenvolver a diabetes gestacional, além dos benefícios adicionais como a melhoria dos resultados observados em sua composição corporal, diminuindo seu percentual de gordura, contribuindo para melhor saúde do feto (Rosa, 2018).

Por fim, há de se destacar algumas limitações do presente trabalho, como o fato de não ter se buscado a temática em outras bases de dados e de o período ter ficado limitado a oito anos. No entanto, o trabalho apresenta informações que reforçam a importância da prática de atividade física e do exercício físico como fator de proteção e estratégia de minimização de prejuízos em mulheres gestantes e em pós parto. 


\section{Considerações Finais}

As recomendações da prática da atividade física em todas as etapas da vida, seja na infância, na vida adulta ou na fase da velhice são frequentemente recomendadas por profissionais da saúde. Entretanto, ao tratarmos de gestantes e mulheres no período pós parto, encontra-se ainda certa resistência à essa prática. Observa-se que a pratica de atividade física e adoção de hábitos saudáveis é fundamental na redução de riscos e prejuízos adversos a saúde da mulher, contribuindo na redução de problemas e agravos no que se refere a integridade física, contribuindo para a manutenção da saúde e a qualidade de vida da mãe e do filho.

O trabalho propôs investigar a efetividades dos exercícios físicos e suas respostas cardiorrespiratórias em mulheres gravidas e em período pós parto. Nota-se grande variedade de estudos que abordam a importância bem como os benefícios do exercício para essa população em tais períodos. Os exercícios resistidos parecem ser uma importante estratégia para a diminuição dos prejuízos causados pelas alterações fisiológicas e psicossociais presentes nestes marcos da vida da mulher.

As melhorias cardiorrespiratórias em virtude do exercício físico é positivo para gestante uma vez que contribui para a saúde da mulher e do bebê, bem como as melhorias cardiorrespiratórias evidenciadas para mulheres na fase pós parto considerando as particularidades deste marco, diminuindo agravos em saúde na fase da maternidade.

A relevância do trabalho parte da necessidade das discussões a partir de informações já disponíveis na literatura a respeito da atuação dos profissionais da saúde, sobretudo, a educação física frente aos cuidados em saúde da mulher gestante, reforçando a importância das diversas contribuições cientificas para que exista a qualificação de recursos humanos para atuar com essa população através da atualização de conhecimento em saúde. Estudos futuros poderiam analisar, de forma prática, a efetividade dos exercícios físicos, verificando em que medida estas estão articuladas com os aspectos teóricos para melhor compreensão sobre a temática.

Por fim, acredita-se que essa temática de grande importância, uma vez que busca abordar sobre respostas para questões que ainda são pouco discutidas na sociedade. Ressalta-se a necessidade dos profissionais da saúde busquem conhecimentos a respeito das prescrições, orientações e contraindicações na realização de atividade física para essa população, para que a intervenção seja realizada de forma eficaz e segura garantindo a saúde da mulher.

\section{Referências}

American College of Obstetricians and Gynecologists (1994). Exercise during pregnancy and the postpartum period. O College.

Costa M., Rocha, L., \& Oliveira S. (2012). Educação em saúde: estratégia de promoção da qualidade de vida na terceira idade. Revista Lusófona de Educação. 22:123-140.

Coutinho, E. de C., et al (2014). Gravidez e parto: O que muda no estilo de vida das mulheres que se tornam mães? Rev. esc. enferm. USP, $48,17-24$.

Estrela, C. (2018). Metodologia Científica: Ciência, Ensino, Pesquisa. Editora Artes Médicas.

Felix, C. A., Nascimento, N. dos S., Almeida, S. C. R. de., \& Oliveira, N. P. C. de. (2021). Pilates method applied to the pregnancy period: its benefits for natural childbirth. Research, Society and Development, 10(14), e593101422495. https://doi.org/10.33448/rsd-v10i14.22495.

Freitas, G. (2017). Enfermagem e a prática de exercícios físicos durante a gravidez. Mindelo.

Lehnen, A. M. et al. (2019). Exercício físico para populações especiais. Sagah.

Mattos, R. A. (2001). Os sentidos da integralidade: algumas reflexões acerca dos valores que merecem ser defendidos. In: Pinheiro R, Mattos RA, editores. Os sentidos da integralidade na atenção e no cuidado à saúde. IMS/ABRASCO, 39-64.

Oliveira, S. S, \& Mattos, J. G. S. (2016). Benefícios da Atividade Física no Período Gestacional. Revista Saúde e Educação, Coromandel, 1(1), 32-44, ago./dez.

Organização Pan-americana de Saúde (2018). Doenças crônico-degenerativas e obesidade: estratégia mundial sobre alimentação saudável, atividade física e saúde. Brasília, DF.

Pereira, J. F., \& Aguiar, V. L. S. (2016). Atividade física e gestação: uma breve revisão da literatura. Vitória. 
Research, Society and Development, v. 11, n. 1, e47311125309, 2022

(CC BY 4.0) | ISSN 2525-3409 | DOI: http://dx.doi.org/10.33448/rsd-v11i1.25309

Rocha, J. F., Rocha, J. R. de. C., \& Costa, J. B. B. (2019). Gestação e exercícios físicos: qualidade de vida para a mãe e o bebê. Revista Diálogos em Saúde. 2(1).

Rosa, L. C. G. (2018). O conhecimento de profissionais de educação física de igrejinha-rs sobre a prescrição do exercício para mulheres no período gestacional. Novo Hamburgo.

Silva, M. E., Silva, W. M. da, Silva, E. R. B., Bezerra, J. J., Costa, J. dos S., Souza, J. N. V. A., Araújo, A. A., Silva, W. N. de S., Couto, S. J. de, Santos, A. B., Barbosa, J. C., Afonso, R. K. de M., Ferreira, A. P. C., Silva, W. K. L. B. da ., Santos, N. F. G. dos, Santos, C. R. N. dos, Souza, K. A. F., \& Almeida, V. M. de L. (2020). Benefits of physical exercise in pregnancy: an integrative review. Research, Society and Development, 9(11), e83291110401. https://doi.org/10.33448/rsd-v9i11.10401. 